\title{
Preparação de sóis aquosos de pseudoboemita com fibrilas de comprimentos diferentes
}

\section{(Preparation of pseudoboehmite aqueous sols with fibril of different lengths)}

\author{
P. S. Santos ${ }^{1}$, A. C. V. Coelho ${ }^{1}$, H. S. Santos ${ }^{2}$, P. K. Kiyohara ${ }^{2}$ \\ ${ }^{1}$ Laboratório de Matérias Primas Particuladas e Sólidos Não Metálicos, Departamento de Engenharia \\ Metalúrgica e de Materiais, Escola Politécnica Universidade de S. Paulo - USP, Av. Prof. Luciano Gualberto, \\ Travessa 3, 380, S. Paulo, SP 05508-970 \\ ${ }^{2}$ Laboratório de Microscopia Eletrônica - LME, Departamento de Física Geral, Instituto de Física - IF, \\ Universidade de S. Paulo - USP, CP 66318, S. Paulo, SP 05315-970 \\ acvcoelh@usp.br
}

\begin{abstract}
Resumo
O mineral boemita, de composição (AlO)OH, na forma sintética pode ter estruturas cristalinas denominadas "boemita bemcristalizada", "pseudoboemita" e "pseudoboemita fibrilar", caracterizáveis por difração de raios X e por microscopia eletrônica de transmissão. É feita uma revisão das propriedades e das aplicações desses tipos de boemita em cerâmica, catalisadores e ab/ adsorventes, enfatizando a especificidade do tipo nas diversas aplicações. É descrito um método de preparação de sóis e géis aquosos de pseudoboemita fibrilar, muito pura, usando matérias-primas nacionais: alumínio em pó e ácido acético, não precisando usar $\mathrm{Hg}^{2+}$ como "ativador" da reação. Controlando o tempo de envelhecimento térmico dos produtos da reação, obtêm-se fibrilas de comprimento médio desde $53 \mathrm{~nm}$ até $720 \mathrm{~nm}$. O método permite também preparar sóis aquosos monofásicos e bifásicos, bem como cristais coloidais de pseudoboemita fibrilar. As fibrilas de pseudoboemita com até 168 h de envelhecimento térmico são "high surface materials" porque sua área de superfície específica é maior que $100 \mathrm{~m}^{2} / \mathrm{g}$.

Palavras-chave: hidróxido de aluminio, boemita, pseudoboemita, pseudoboemita fibrilar, pseudoboemita coloidal.
\end{abstract}

\begin{abstract}
The mineral boehmite, of composition (AlO)OH, as a synthetic material may have crystalline structures named "well-crystallized boehmite", "pseudobehmite", and "fibrillar pseusoboehmite", characterized by X-ray diffraction and transmission electron microscopy. A review is made of the properties and applications of these boehmite types in ceramics, catalysts and ab/adsorvents, calling attention to the specificity of the structure type in the several applications. A method is described to prepare aqueous sols and gels of fibrillar pseudoboehmite, very pure, using Brazilian raw materials: aluminum powder and acetic acid, avoiding Hg ${ }^{2+}$ as "activator" of the reaction. Controlling the thermal ageing time of the reaction products, fibrils are obtained with average length from $53 \mathrm{~nm}$ to $720 \mathrm{~nm}$. The method also allows the preparation of monolayer and double-layer sols, as well as colloidal crystals of fibrillar pseudoboehmite. The pseudoboehmite fibrils up to 168 h of thermal ageing are "high surface materials", because its specific surface area is larger than $100 \mathrm{~m}^{2} / \mathrm{g}$.
\end{abstract}

Keywords: aluminum hydroxide, boehmite, pseudoboehmite, fibrillar pseudoboehmite, colloidal pseudoboehmite.

\section{INTRODUÇÃO}

Boemita é um mineral de hidróxido de alumínio, de composição $\mathrm{AlOOH}$, componente de bauxitos cársticos [1] e de algumas argilas altamente aluminosas [2]. Gitzen, da ALCOA, cita entre as conclusões de um simpósio de 1957 sobre normatização de nomenclaturas que, no caso do alumínio, hidróxido deve substituir hidrato [3]; assim, deve ser trihidróxido de alumínio em lugar de alumina trihydrate, e óxido-hidróxido de alumínio em lugar de alumina monohydrate. O monohidróxido de alumínio ou óxido-hidróxido de alumínio sintético, de fórmula (AlO)OH ou $\mathrm{Al}_{2} \mathrm{O}_{3} \cdot \mathrm{H}_{2} \mathrm{O}$, pode ter a estrutura cristalina do mineral boemita. Lippens e Sterggerda [4] distinguem dois sólidos: boemita bem-cristalizada ("well-crystallized boehmite", boemita $\mathrm{BC}$ ) e pseudoboemita ou boemita gelatinosa.

A boemita $\mathrm{BC}$ é caracterizada por um difratograma de raios $\mathrm{X}$ com reflexões agudas e intensas e um valor de reflexão basal (020) $\mathrm{d}_{020}=6,11 \AA=0,61 \mathrm{~nm}[5]$; os dados de boemita BC estão registrados no arquivo 21-1307/ Boemita do ICDD. A Fig. 1A é o difratograma de raios X da boemita BC sintetizada hidrotermicamente por $8 \mathrm{~h}$. A Fig. 


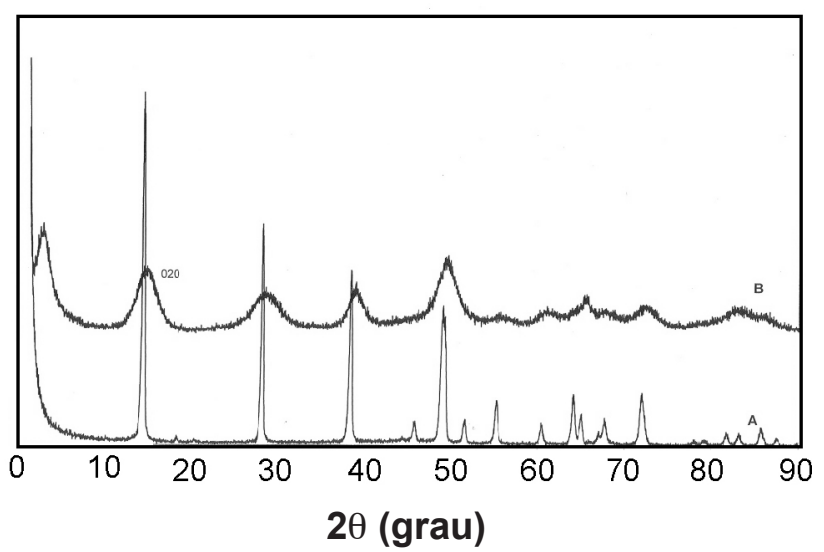

Figura 1: A) Difratograma de raios X de boemita bem-cristalizada de síntese hidrotérmica; B) de uma pseudoboemita mostrando o alargamento das bandas de difração nas mesmas posições da boemita BC.

[Figure 1: A) X-ray diffraction patterns of a comercial wellcrystallized boehmite, from hydrothermal synthesis; B) of a pseudobehmite, showing the line broadenings at the same positions of the wc-boehmite.]

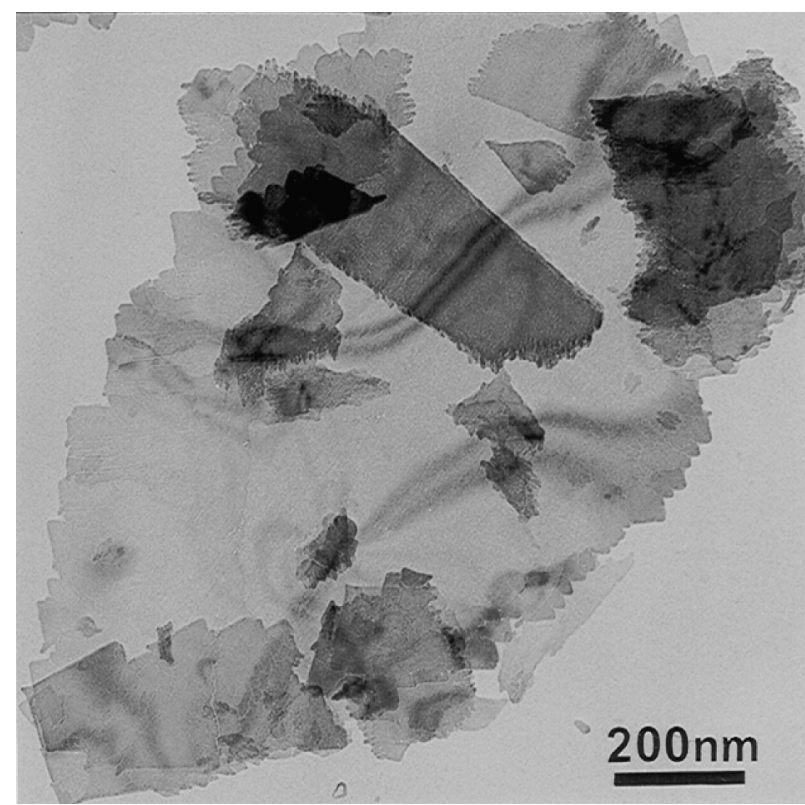

Figura 2: Micrografia obtida por microscopia eletrônica de transmissão dos cristais de boemita BC da Fig. 1A com formato lamelar e perfil irregular.

[Figure 2: Transmission electron microscopy micrograph of wcboehmite crystals from Fig. $1 \mathrm{~A}$ with platy shape and irregular profile.]

2 é uma micrografia eletrônica de transmissão mostrando a morfologia lamelar dos cristais de boemita $\mathrm{BC}$ sintéticos.

Papée et al. [6] assinalaram os fatos de que os hidróxidos de alumínio sintéticos, que foram denominados pseudoboemita, além de terem as reflexões nas mesmas posições da boemita BC, essas reflexões eram menos intensas e as curvas alargadas. Mais tarde, Krivoruchko et al. [7] propuseram a seguinte definição para pseudoboemita: “entende-se por pseudoboemita o hidróxido de alumínio com desordem na estrutura cristalina ("poorly crystallized"), de composição $\mathrm{Al}_{2} \mathrm{O}_{3} \cdot \mathrm{xH}_{2} \mathrm{O}(2,0>\mathrm{x}>1,0)$, com distâncias interplanares aumentando na direção $\{020\}$ até o valor de $6,7 \AA$, em comparação com $6,11 \AA$ para boemita". Após uma longa controvérsia entre uma dezena de pesquisadores, Tettenhost e Hoffmann [8] concluíram, após o estudo de 32 amostras de boemitas preparadas por vários métodos, que as diferenças apresentadas nos difratogramas de raios $\mathrm{X}$ dessas boemitas podem ser explicadas, principalmente, por variações nos tamanhos dos cristalitos; portanto, segundo eles, boemita $\mathrm{BC}$ e pseudoboemita são contínuos na sua estrutura e propriedades físicas. A Fig. 1B é um difratograma de raios $\mathrm{X}$ de pseudoboemita, mostrando o alargamento das reflexões características em comparação com as de boemita BC da Fig. 1A.

Souza Santos et al. [9] mostraram, por microscopia eletrônica de transmissão e por difração eletrônica, que hidróxido de alumínio não-cristalino ou amorfo (por difração de raios $\mathrm{X}$ ), em meio aquoso, aquecido a $70{ }^{\circ} \mathrm{C}$ por $2 \mathrm{~h}$, transforma-se em microfibrilas, cujos anéis de difração correspondiam aos de pseudoboemita. Bugosh patenteou nos EUA [10] sob o nome de "Baymal" um material que chamou "fibrous alumina monohydrate"; era obtido a partir do produto da reação entre soluções aquosas de cloreto de alumínio e hidróxido de sódio, aquecidos entre $120{ }^{\circ} \mathrm{C}$ e $250{ }^{\circ} \mathrm{C}$. Em outras publicações, Bugosh denominou também "fibrous boehmite", "colloidal boehmite", "fibrillar boehmite"; Iler [11] denominou "fibrillar colloidal boehmite"; Abrams e Low [12], em trabalho sobre "fibrillar synthetic boehmite

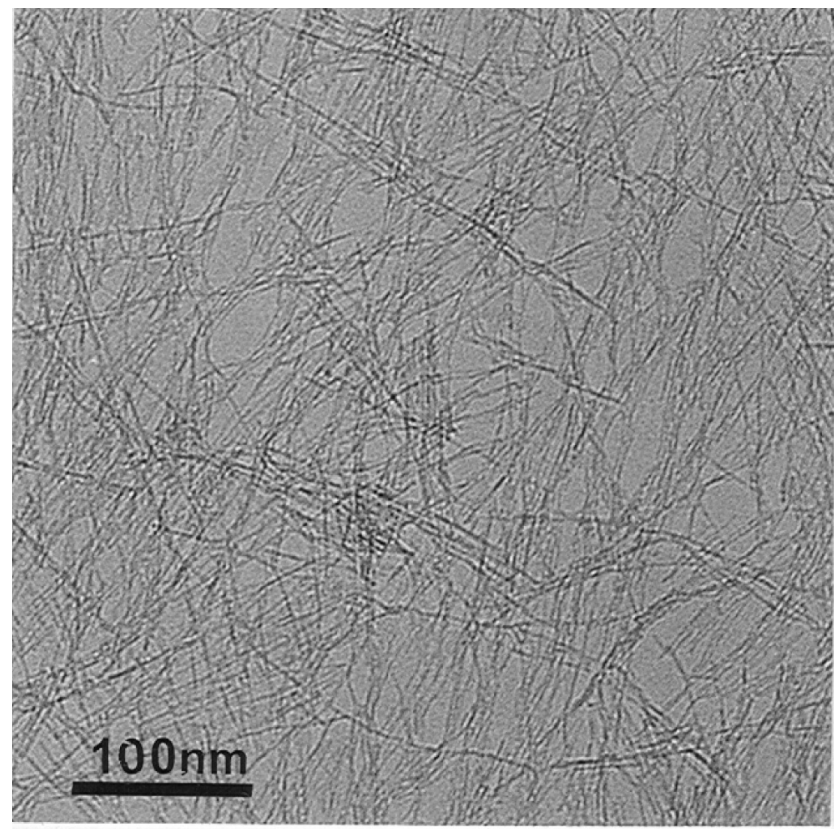

Figura 3: Micrografia obtida por microscopia eletrônica de transmissão de fibrilas de pseudoboemita, mostrando que são flexíveis e as extremidades não são ponteagudas.

[Figure 3: Transmission electron microscopy micrograph of "pseudoboehmite" fibrils, showing that they are flexible and their extremities are not pointed.] 


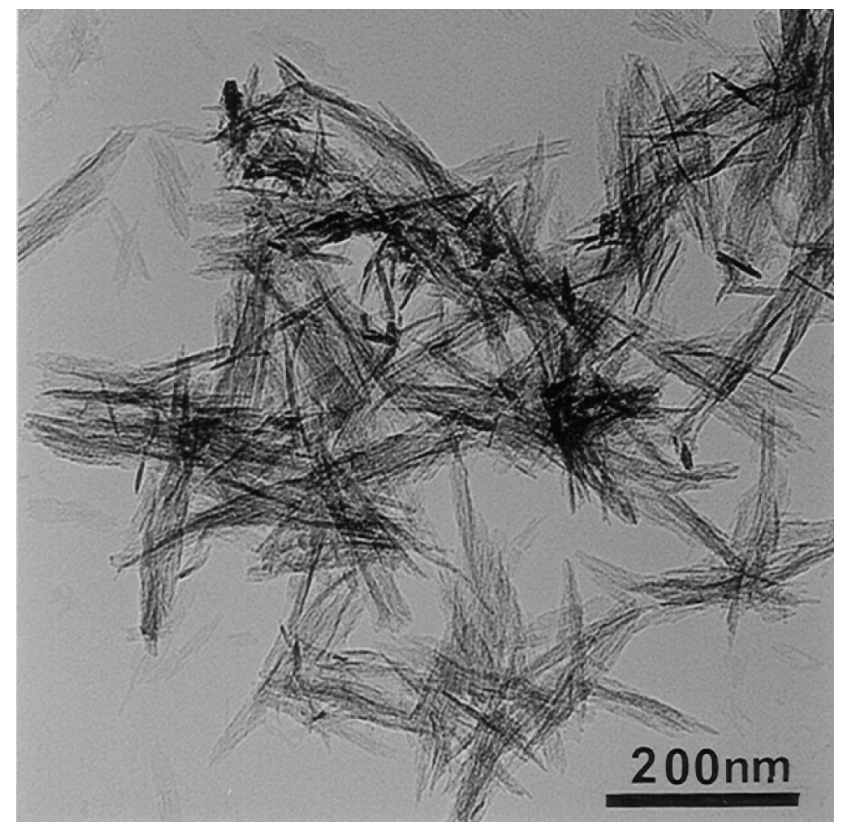

Figura 4: Micrografia obtida por microscopia eletrônica de transmissão de feixes de fibrilas de pseudoboemita, com aspecto acicular, preparada por precipitação homogênea de solução de nitrato de $\mathrm{Al}+$ uréia.

[Figure 4: Transmission electron microscopy micrograph of fibrils bundles of pseudoboehmite, having needle-like shape, from homogeneous precipitation of aluminum nitrate and urea solution.]

Baymal", considera, com base no difratograma de raios X, "not highly crystalline"; portanto, é uma pseudoboemita fibrilar. Misra [13] escreve que Baymal consiste de "minute fibrils of boehmite". O produto comercial Versal é um gel de pseudoboemita constituído por fibrilas [14]. Existem outros produtos comerciais em que os microcristais de boemita são descritos como "needles-like", "tiny needles", acicular", "thin acicular grains". Aparentemente, existe uma diferença entre pseudoboemita fibrilar e pseudoboemita acicular: os cristais são flexíveis nas fibrilas e são rígidos e tem pelo menos uma ponta nas agulhas. A Fig. 3 mostra a micrografia obtida por microscopia eletrônica de transmissão de cristais de pseudoboemita fibrilar e a Fig. 4 é de pseudoboemita acicular. A variedade de morfologia dos cristais dos diferentes tipos de boemita, conforme o método de síntese, foi descrita por Souza Santos et al. [15 ].

Usos cerâmicos e não-cerâmicos de boemita - Produtos refratários de alta alumina podem ter boemita $\mathrm{BC}$ como ligante a partir de alumina tabular (alumina-alfa) e alumina reativa A-16SG autoclavados em vapor d'água saturado a 190-240 ${ }^{\circ} \mathrm{C}$ [16]. Whiskers de alumina-alfa podem ser crescidos a partir de géis aquosos de pseudoboemita [17]. Aluminagama, a partir de boemita foi escolhida por Sappei et al. [18] como precursor para síntese de AlON porque é isoestrutural com AlON-gama. Boemita coloidal tem sido amplamente utilizada na tecnologia solgel [19], boemita coloidal é usada como agente plastificante e ligante para pastas dilatantes de alumina-alfa e água [20-22]. Pierre e Ulmann [23] apontaram as analogias de propriedades físico-químicas entre solgéis de argilas (bentonitas-Na) e solgéis aquosos de boemita: Pós de boemita/pseudoboemita desempenham papéis importantes na preparação de aluminas e de materiais cerâmicos derivados de alumina, com porosidade, permeabilidade, resistência mecânica e propriedades elétricas desejadas [24, 25]. Sol aquoso de boemita é um dos materiais candidatos ideais para a fabricação de componentes cerâmicos à base de alumina de alta qualidade, com microestruturas de sinterização final controlada, porque contem partículas cristalinas facilmente sinterizáveis, com dimensões na escala nanométrica [26, 27].

Boemita é o único hidróxido de alumínio cristalino que, por uma reação química direta de desidroxilação, produz a alumina de transição alumina-gama, a partir do aquecimento a $450{ }^{\circ} \mathrm{C}$; a $1100{ }^{\circ} \mathrm{C}$, a seqüência é $\mathrm{AlOOH}-\gamma-\delta-\theta-\alpha \mathrm{Al}_{2} \mathrm{O}_{3}$; as transformações boemita- $\gamma, \gamma-\delta, \delta-\theta$ são topotáticas $\mathrm{e}$ pseudomórficas a partir da morfologia original do cristal de boemita [11, 28-35]

Pseudoboemita é excelente material ligante para partículas ou cristais de catalisadores e ab/adsorventes que, sozinhos, não tem capacidade para ser conformados em peças com formas e dimensões variadas. Alumina-gama (com área específica $\left.150 / 300 \mathrm{~m}^{2} / \mathrm{g}\right)$; alumina-delta $\left(100 / 150 \mathrm{~m}^{2} / \mathrm{g}\right)$ e alumina-theta $\left(100 / 150 \mathrm{~m}^{2} / \mathrm{g}\right)$ são utilizadas como catalisadores, suportes de catalisadores e ab/adsorventes [36].

Alumina-gama é importante como suporte de catalisadores porque a forma acircular ("tiny needles") das partículas facilita o recobrimento por precursores dos catalisadores [37], além de manter uma área de superfície específica elevada, acima de $100 \mathrm{~m}^{2} / \mathrm{g}$ até $1000^{\circ} \mathrm{C}$ [38]. Membranas de alumina-gama, com porosidade em nanoescala, além de serem utilizadas em processos de separação, podem ser também usadas como membranas catalíticas em reatores químicos, como por exemplo para a reforma de $\mathrm{CH}_{4}$ em $\mathrm{CO}+\mathrm{H}_{2}$ [39-41].

Boemita sintética foi um dos primeiros materiais a serem usados como precursores de alumina-alfa no processo solgel, devido aos processos de síntese gerarem cristais com dimensões coloidais ou nanométricas (isto é, entre 1 $\mathrm{nm}$ e $100 \mathrm{~nm}$ ), e serem facilmente dispersáveis em água por ácidos como $\mathrm{HNO}_{3}$, produzindo sóis e géis estáveis, com concentrações elevadas, de até $20 \%$ [30, 42-50].

Atualmente, existem boemitas sintéticas comerciais, em pós (nanométricos) e sóis aquosos concentrados. Os vários tipos foram descritos por Misra [13]. Examinando a maioria das publicações dos últimos 40 anos, envolvendo boemita, de um modo geral observa-se que são utilizados produtos comerciais ou então é feita a síntese em laboratório. Neste último caso, três linhas são usadas para produzir hidróxido de alumínio não-cristalino, em meio aquoso, o qual é aquecido entre $60^{\circ} \mathrm{C}$ até a ebulição, por tempos variando de $2 \mathrm{~h}$ a 3 dias, porém de forma independente. Exemplificando: $2 \mathrm{~h}$ a $86{ }^{\circ} \mathrm{C}$ ou 3 dias a $60{ }^{\circ} \mathrm{C}$; essas condições de preparação, se a temperatura for inferior a $130{ }^{\circ} \mathrm{C}$, leva à formação de pseudoboemita fibrilar, cujas fibrilas tem comprimentos que aumentam com o aumento da temperatura e do tempo [31, 35]. Em quase todos os trabalhos analisados há controle por difração de raios $\mathrm{X}$, com diagnóstico, por exemplo, 
se é boemita $\mathrm{BC}$ ou pseudoboemita. É raro o caso em que há controle da morfologia dos cristais por microscopia eletrônica de transmissão: "needle-like" [45], acicular [18], "tiny needles" [37], "rod-like" [26], "thin plates" [19, 28], "platelets" [19, 23, 39]. Esse fato é curioso porque tanto nessas publicações como naquelas citadas anteriormente, sobre a natureza da pseudoboemita, como o artigo de Tettenhorst e Hofmann [8], o pequeno tamanho dos cristais é apontado como o fator principal das diferenças entre boemita $\mathrm{BC}$ e pseudoboemita, sem se importar o efeito da morfologia dos cristais sobre as propriedades dos pós e dos sistemas coloidais [51-53]

O objetivo deste trabalho é o de descrever um método de preparação de sóis aquosos concentrados constituídos por fibrilas de pseudoboemita com comprimentos diferentes.

\section{MATERIAIS E MÉTODOS}

Alumínio em pó: foi utilizada amostra da ALCOA do Brasil tipo 123; composição química: Al 99,84\%, Si 0,04\%, Fe 0,09\%; granulometria 96,6\% abaixo da peneira ABNT 325 (abertura $44 \mu \mathrm{m}$ ), fabricado em Poços de Caldas, MG.

A reação química foi conduzida em um balão pyrex de 2 L com 3 bocas, aquecido em manta elétrica, equipado por condensador para refluxo resfriado a água, agitador mecânico contínuo, e termômetro com termostato acoplado à manta de aquecimento.

Para a preparação foram utilizadas $27,0 \mathrm{~g}$ de alumínio em pó. Foi empregada uma relação molar $1 \mathrm{Al}: 50 \mathrm{H}_{2} \mathrm{O}$, a mesma utilizada em estudos dos estágios iniciais de polimerização do hidróxido de alumínio não-cristalino [54, 55]. Foi utilizada a proporção $1 \mathrm{Al}: 0,5 \mathrm{CH}_{3} \mathrm{COOH} \cdot 50 \mathrm{H}_{2} \mathrm{O}$ para a produção dos monômeros $\mathrm{Al}(\mathrm{OH})_{3}$ não-cristalinos e $\mathrm{OH}-\mathrm{Al}-\mathrm{Ac}$ (Ac é $\mathrm{CH}_{3} \cdot \mathrm{COO}^{-}$); proporções superiores a $0,5 \mathrm{HAc}: 1 \mathrm{Al}$ levam à formação do composto cristalino insolúvel $\mathrm{Al}(\mathrm{OH}) \mathrm{Ac}_{2}$, que precipita. A relação $1 \mathrm{Al}: 50 \mathrm{H}_{2} \mathrm{O}$ leva a uma concentração final de pseudoboemita nos sóis de $\sim 70 \mathrm{~g} / \mathrm{L}$, expressa em $\mathrm{AlOOH}$.

A seqüência de adição dos reagentes é: água, todo o alumínio e todo HAc de uma vez. Inicia-se a agitação (1800 $\mathrm{rpm})$ e o aquecimento da manta até o termômetro marcar 90 $( \pm 2)^{\circ} \mathrm{C}$. O aquecimento é continuo, sob refluxo, mantendo constante o volume do sol. O tempo de envelhecimento térmico a $90{ }^{\circ} \mathrm{C}$ estendeu-se até $1000 \mathrm{~h}$. Obtém-se 900-920 $\mathrm{mL}$ do sol por batelada nas preparações até $168 \mathrm{~h}$.

Foram pipetadas alíquotas de $10 \mathrm{~mL}$ após os seguintes tempos de aquecimento (h): $0,8,16,24,36,48,60,72$, 84, 96, 120, 144, 168, 192, 300, 400, 500 e 1000. Essas alíquotas foram usadas para caracterização estrutural por difração de raios X e morfologia por microscopia eletrônica de transmissão.

As alíquotas foram colocadas em moldes de poliestireno cilíndricos com diâmetro $10 \mathrm{~cm}$ e altura $0,5 \mathrm{~cm}$, foram secas à temperatura ambiente com circulação de ar. $\mathrm{O}$ sol forma, após secagem, membranas rígidas. Até $36 \mathrm{~h}$, trincam e se fragmentam. Após esse tempo são continuas, sem defeitos. Foram moídas em almofariz e passadas totalmente em peneira
ABNT $200(74 \mu \mathrm{m})$. O pó foi examinado em difratômetro de raios X Philips X'Pert MPD com radiação $\mathrm{k}_{\text {alfa }}$ do cobre operando entre $2 \theta=1^{\circ}$ e $90^{\circ}$. A caracterização do hidróxido de alumínio boemita ou pseudoboemita foi feita com base em [5] e nos arquivos do ICDD.

Uma gota de cada alíquota foi diluída em $50 \mathrm{~mL}$ de água destilada e preparada para o exame no microscópio eletrônico de transmissão pelo procedimento convencional. Foi utilizado um microscópio Philips CM200 operando a 200 kV. Quando necessário, o microscópio eletrônico de transmissão foi usado como câmara de difração eletrônica. O mesmo foi feito com o microscópio eletrônico de varredura.

\section{RESULTADOS E DISCUSSÃO}

\section{Difração de raios $X$}

Todos os pós provenientes das alíquotas com $36 \mathrm{~h}$ ou mais de aquecimento a $90{ }^{\circ} \mathrm{C}$ apresentaram valores de $d_{020}$ superiores a $0,611 \mathrm{~nm}$ e inferiores a $0,67 \mathrm{~nm}$. As reflexões são largas e estão nas posições correspondentes às da boemita bem-cristalizada. Alguns difratogramas de raios $\mathrm{X}$ de pós de alíquotas de um mesmo sol, com tempos crescentes de aquecimento, estão mostrados na Fig. 5, em comparação com os de boemita bem-cristalizada, que é a mesma da Fig. 1A. Nota-se um aumento do afinamento dos picos com o aumento do tempo de envelhecimento de $24 \mathrm{~h}$ a $192 \mathrm{~h}$; esse fato poderia ser interpretado, quer como devido ao crescimento dos monocristais, quer como aumento da ordem estrutural ou grau de cristalinidade. Os valores medidos para os difratogramas de raios X da Fig. 5 para $\mathrm{d}_{020}$ são: 48 h-0,64 $\mathrm{nm}, 72 \mathrm{~h}-0,638 \mathrm{~nm}, 96 \mathrm{~h}-0,637 \mathrm{~nm}, 120 \mathrm{~h}-0,637 \mathrm{~nm}, 144$ h-0,637 nm, 168 h- $0,633 \mathrm{~nm}$, e 192 h-0,633 nm; também para $500 \mathrm{~h}-0,627 \mathrm{~nm}$ e para $1000 \mathrm{~h}-0,615 \mathrm{~nm}$; nessa temperatura e nesse tempo de envelhecimento não foi encontrado o valor de $\mathrm{d}_{020}=0,611 \mathrm{~nm}$ de boemita bem-cristalizada. Como pode ser observado na Fig. 5, todas as reflexões de 24 h a 192 h estão alargadas em comparação com os picos agudos de boemita bem-cristalizada. Portanto, todas as curvas de 48 h a 192 h são de pseudoboemita. Nas alíquotas com até 24 $\mathrm{h}$ de aquecimento, como pode ser observado na Fig. 5A, não ocorre a reflexão de 0,6 a $0,7 \mathrm{~nm}$, correspondente ao plano (020); entretanto, os pós examinados por microscopia eletrônica de transmissão são fibrilas curtas que apresentam, por difração eletrônica, reflexões (anéis de difração) nas posições de boemita. Portanto, as partículas dos sóis até 24 h são constituídas por pseudoboemita do tipo "amorfo à difração de raios X", mas "cristalino à difração de elétrons", devido às pequenas dimensões dos cristais [56].

Observa-se também na Fig. 5, nos difratogramas de raios $\mathrm{X}$ de $48 \mathrm{~h}$ a $192 \mathrm{~h}$, uma reflexão na região de pequenos ângulos, $2,5^{\circ}$ a $2,1^{\circ}(2 \theta)$ - com valor de $\sim 35 \AA-36 \AA$; esse pico foi encontrado nos sóis de 135 preparações estudadas, inclusive em sóis com $1000 \mathrm{~h}$ de envelhecimento a $90{ }^{\circ} \mathrm{C}$. Brindley e Simonton [57] observaram picos na região de $1^{\circ}(2 \theta)$ para géis úmidos e secos dos argilo-minerais parigorsquita 


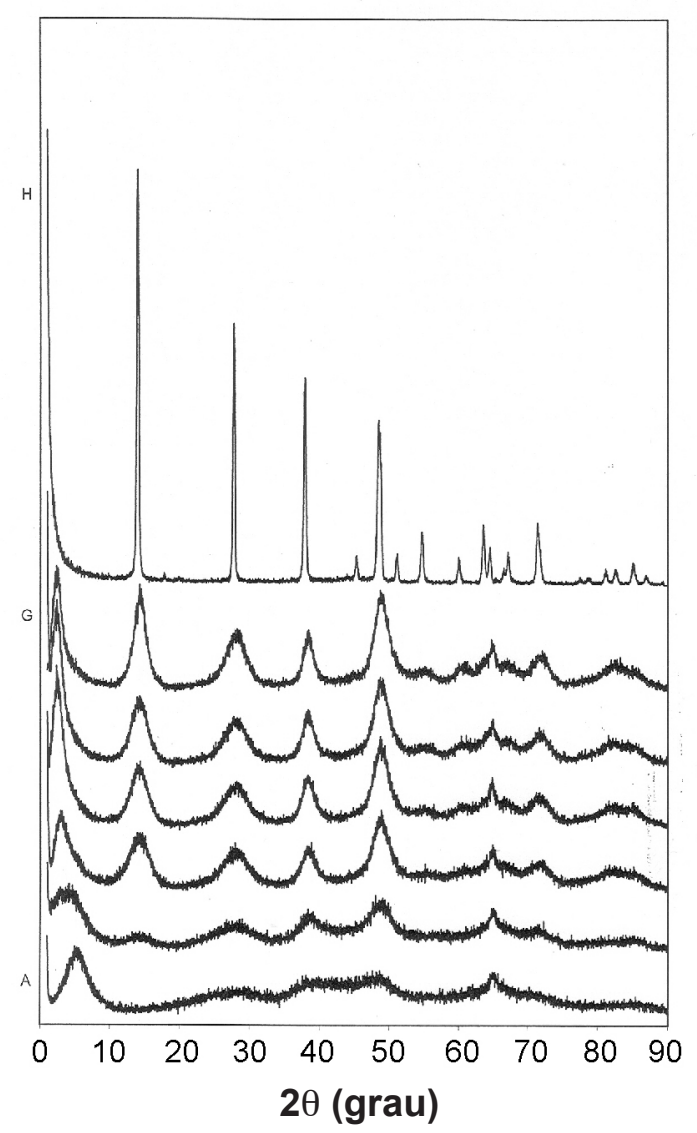

Figura 5: (A) até (G) - Difratogramas de raios $\mathrm{X}$ de alíquotas de um mesmo sol de pseudoboemita fibrilar com 8 h até 192 $\mathrm{h}$ de aquecimento a $90^{\circ} \mathrm{C}$; $(\mathrm{H})$ da boemita $\mathrm{BC}$ da Fig. 1A. [Figure 5: (A) to $(G) X$-ray diffraction patterns of aliquots from the same fibrillar pseudoboehmite sol from $8 \mathrm{~h}$ up to $192 \mathrm{~h}$ of thermal aging at $90{ }^{\circ} \mathrm{C},(\mathrm{H})$ of wc-boehmite, the same as in Fig. 1A.]

e sepiolita, cujos microcristais são ripas alongadas com morfologia e dimensões semelhantes às das fibrilas de pseudoboemita. A origem desse pico a $\sim 35 \AA$ A-36 ̊s será discutida em publicação sobre a cristalização hidrotérmica da pseudoboemita fibrilar.

\section{Microscopia eletrônica de transmissão}

Excetuando-se a alíquota de $0 \mathrm{~h}$, todas as outras de 8 h a 192 h da Fig. 5, são constituídas por fibrilas flexíveis. Considerando esses resultados e os difratogramas de raios $\mathrm{X}$, considera-se que as alíquotas do sol são constituídas por microcristais de pseudoboemita fibrilar. Os comprimentos das fibrilas das alíquotas da Fig. 5 foram medidas em ampliações com aumento de $70 \mathrm{kX}$, seguindo os seguintes critérios; a) 40 a 50 fibrilas foram medidas por alíquota; b) as duas extremidades da fibrila devem ser bem nítidas; c) a fibrila medida deve ter pequena ou nenhuma curvatura. Os valores da média aritmética dos comprimentos das fibrilas são os seguintes: $24 \mathrm{~h}-0,053$ $\mu \mathrm{m}=53 \mathrm{~nm}, 48 \mathrm{~h}=71 \mathrm{~nm}, 72 \mathrm{~h}=171 \mathrm{~nm}, 96 \mathrm{~h}=360 \mathrm{~nm}, 120$ $\mathrm{h}=371 \mathrm{~nm}, 144 \mathrm{~h}=380 \mathrm{~nm}, 168 \mathrm{~h}=450 \mathrm{~nm}$, e $192 \mathrm{~h}=720$ $\mathrm{nm}$. Segundo Tchoubar e Oberlin [58] as fibrilas crescem na direção do eixo cristalográfico $\mathrm{c}$ da boemita. Algumas observações sobre a evolução das fibrilas: após $96 \mathrm{~h}$ de envelhecimento, nota-se a existência de um grande número de feixes de fibrilas alinhadas paralelamente ao longo dos seus comprimentos; com 168 h, o número de feixes é maior, porém o número de fibrilas (ou o diâmetro do feixe) é muito variável. Os sóis com 300 h, 500 h e 1000 h continuam constituídos por fibrilas de pseudoboemita; um gel úmido, mantido fechado por 15 anos à temperatura ambiente mantém a estrutura e a morfologia original de pseudoboemita fibrilar; aquecimento de sóis e géis de pseudoboemita fibrilar, em meio aquoso e sem aditivos, por mais de $8 \mathrm{~h}$ acima de $80{ }^{\circ} \mathrm{C}$ ou hidrotermicamente entre $100{ }^{\circ} \mathrm{C}$ e $150{ }^{\circ} \mathrm{C}$, não só consolida a formação de pseudoboemita fibrilar, como torna o sistema sol ou gel estável quanto ao envelhecimento e evita a recristalização espontânea em meio aquoso em trihidróxidos de alumínio cristalinos [9, 59-63].

O diâmetro das fibrilas pode ser medido por microscopia

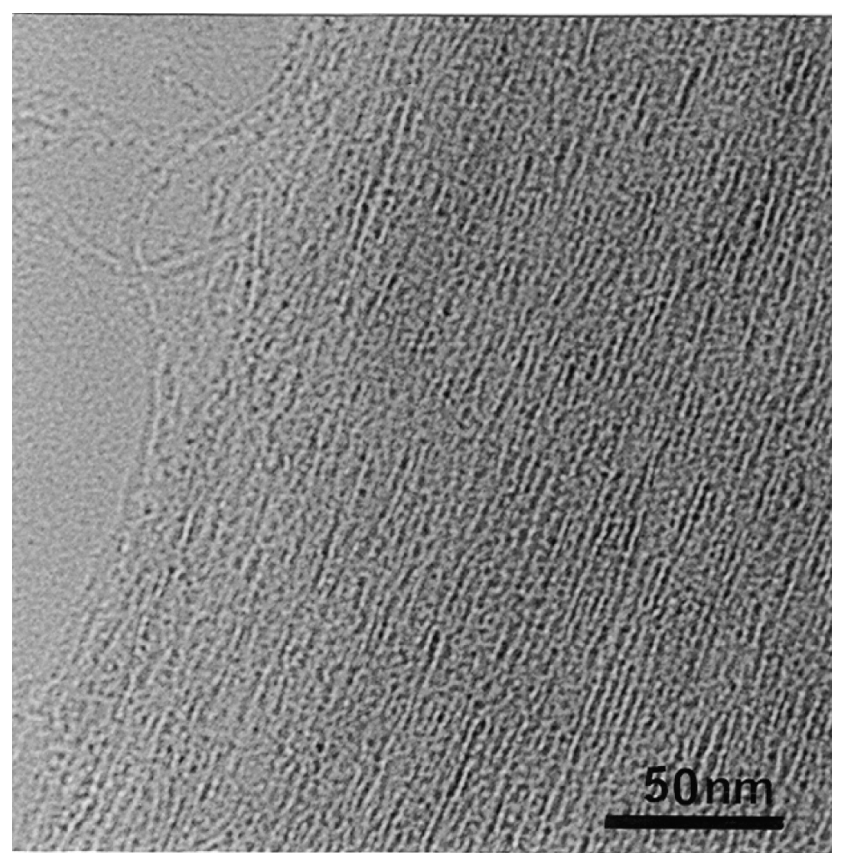

Figura 6: Micrografia obtida por microscopia eletrônica de transmissão de camada monofibrilar de "Pseudoboemita" aumentada 420.000X.

[Figure 6: Transmission electron microscopy micrograph of monolayer of fibrils of "pseudoboehmite" enlarged 420,000X.]

eletrônica de transmissão em camadas monofibrilares, como mostrada na Fig. 6, com aumento de $420 \mathrm{kX}$; medindo 10 fibrilas paralelas tem-se $15 \mathrm{~mm}$; o diâmetro médio é $(15 / 4,2) \mathrm{nm}=3,57 \mathrm{~nm}=35,7 \AA$. Esse valor é da mesma ordem de grandeza do diâmetro de $30 \AA$ medido por Tchoubar e Oberlin [58] em fibrilas de pseudoboemita 
formadas na alteração de feldspato albita pela água e dos picos da ordem de $35 \AA-36 \AA$, mostrados na Fig. 5.

Tipos de sois de "pseudoboemita fibrilar" formados no método descrito

Utilizando o método de preparação descrito e a concentração de $70 \mathrm{~g} / \mathrm{L}$ de $\mathrm{AlOOH}$, três "tipos" de sóis aquosos podem ser preparados, variando o tempo de envelhecimento térmico e mantendo o aquecimento a $90^{\circ} \mathrm{C}$.

a) sóis monofásicos, isto é, que deixados em repouso apresentam apenas uma fase liquida; são preparados com o tempo de envelhecimento térmico entre $8 \mathrm{~h}$ e $60 \mathrm{~h}$. Todos os sóis são tixotrópicos e apresentam forte ditindalismo $[64,65,77]$ ou efeito sedoso, por serem constituídos por fibrilas. Os sóis de até $24 \mathrm{~h}$ deixados em repouso gelificam após 2 semanas. A Fig. 7 apresenta uma micrografia obtida por microscopia eletrônica de transmissão de fibrilas curtas de um sol de $48 \mathrm{~h}$.

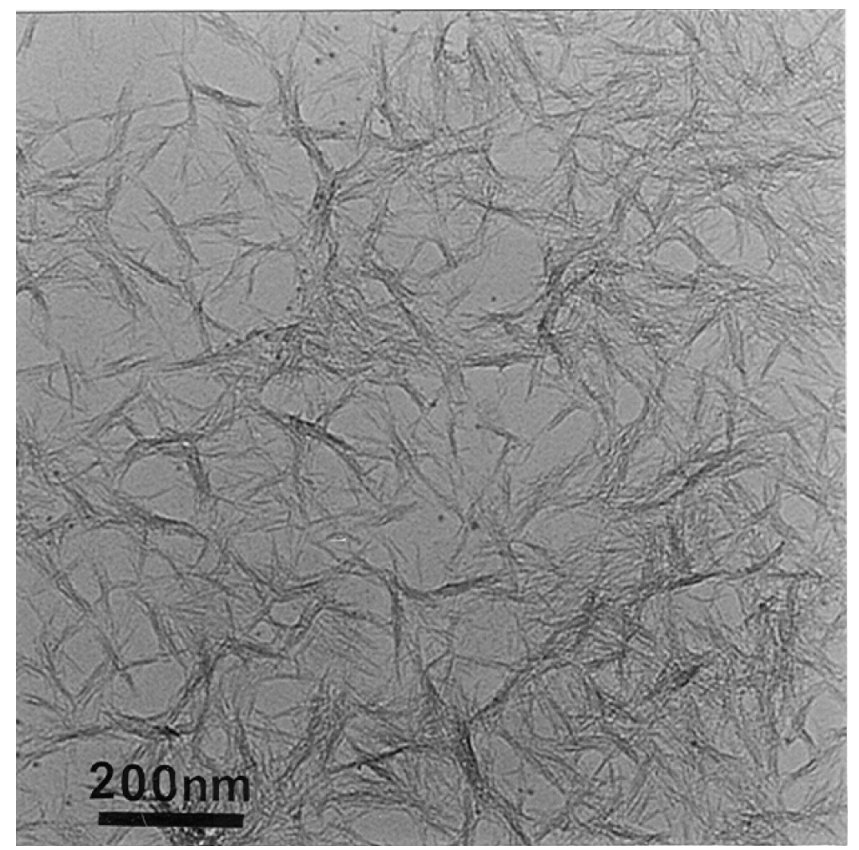

Figura 7: Micrografia obtida por microscopia eletrônica de transmissão de fibrilas curtas de pseudoboemita de um sol com $48 \mathrm{~h}$ de aquecimento a $90{ }^{\circ} \mathrm{C}$ (DRX: Fig. 5B).

[Figure 7: Transmission electron microscopy micrograph of short fibrils of pseudoboehmite from a sol with $48 \mathrm{~h}$ of thermal aging at $90^{\circ} \mathrm{C}$; XRD: Fig. 5B.]

b) sóis bifásicos, isto é, que após repouso por algumas horas, separam-se espontaneamente em duas fases líquidas, miscíveis; são preparados com tempo de envelhecimento de $72 \mathrm{~h}$ a $192 \mathrm{~h}$. A camada ou fase superior pode ter de 55 a $62 \mathrm{~g} / \mathrm{L}$ de $\mathrm{AlOOH}$ e a inferior de 82 a $87 \mathrm{~g} / \mathrm{L}$, variando com o tempo de envelhecimento. $\mathrm{O}$ sistema bifásico pode ser homogeneizado por agitação em um sol instável, com concentração original de $70 \mathrm{~g} / \mathrm{L}$; deixado em repouso, retorna o sol bifásico original. Ambas as fases apresentam forte ditindalismo; a fase superior é isotrópica e a inferior é birrefringente ou anisotrópica. As Figs. 8 e 9 são MET's de fibrilas, respectivamente, da fase superior e da inferior de

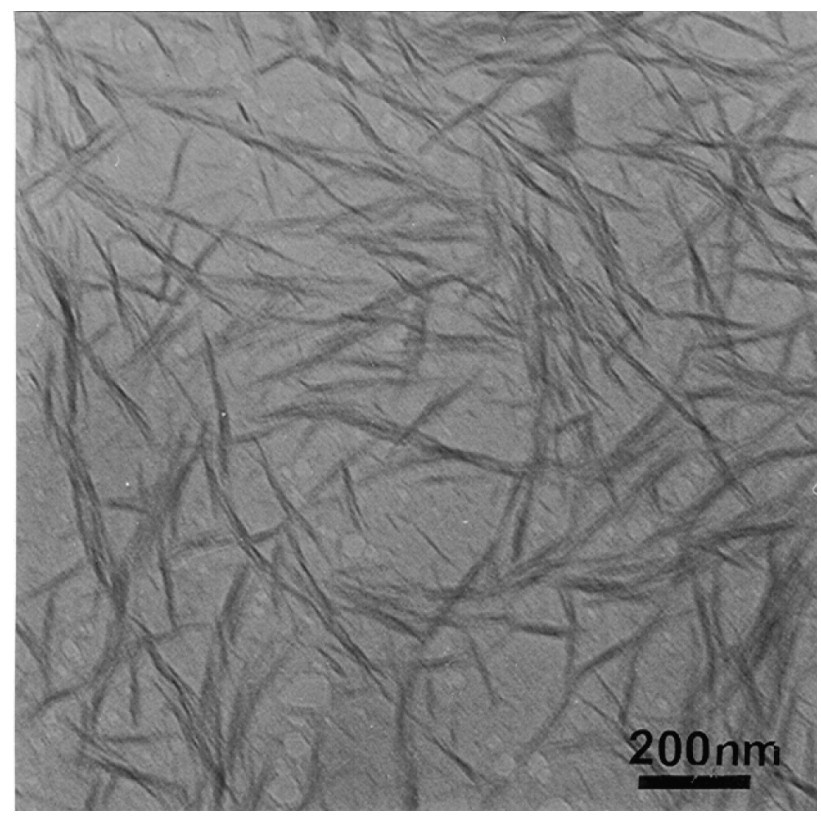

Figura 8: Micrografia obtida por microscopia eletrônica de transmissão da camada superior de um sol bifásico de pseudoboemita fibrilar com $72 \mathrm{~h}$ de envelhecimento térmico a $90{ }^{\circ} \mathrm{C}$.

[Figure 8: Transmission electron microscopy micrograph of the upper layer of the double-layer sol of fibrilar pseudoboehmite with $72 \mathrm{~h}$ of thermal aging at $90^{\circ} \mathrm{C}$.]

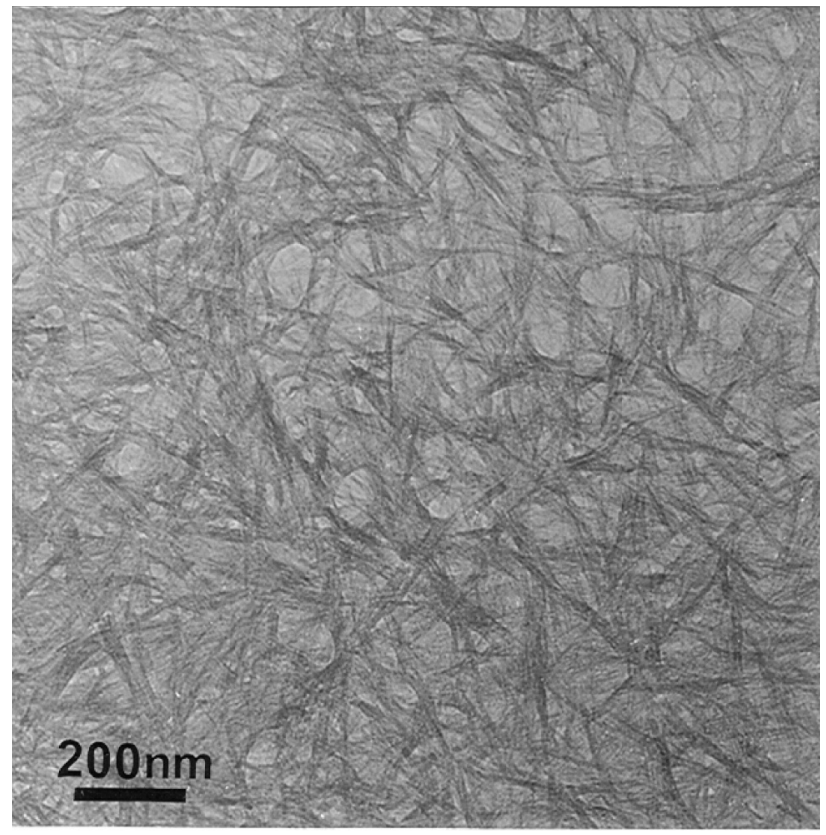

Figura 9: Micrografia obtida por microscopia eletrônica de transmissão da camada inferior do mesmo sol bifásico da Fig. 8. [Figure 9: Transmission electron microscopy micrograph of the bottom layer of the same double-layer sol of Fig. 8.]

um sol com $72 \mathrm{~h}$ de envelhecimento térmico.

c) sistema coloidal trifásico, constituído por duas fases (sóis) liquida e uma fase sólida (cristal coloidal). Esses sistemas formam-se ao se continuar o aquecimento a $90{ }^{\circ} \mathrm{C}$ 
acima de $192 \mathrm{~h}$ até $500 \mathrm{~h}$; observa-se sempre nas alíquotas deixadas em repouso a formação de duas fases líquidas miscíveis, mas reversivelmente e também o aparecimento de um anel e, depois, de um disco flexível de um material sólido insolúvel, de cor branca, com espessura de $\sim 1$ $\mathrm{mm}$; essa espessura aumenta com o decorrer do tempo de envelhecimento. O material sólido, de cor branca, examinado por difração de raios $\mathrm{X}$ e por microscopia eletrônica de transmissão mostrou ser constituído por pseudoboemita fibrilar; examinando por microscopia eletrônica de varredura, mostrou que as fibrilas se alinham paralelamente entre si e tridimensionalmente, formando o sólido com a estrutura cristalina de um cristal coloidal. A Fig. 10 mostra uma micrografia obtida por microscopia eletrônica de varredura de um fragmento desse cristal coloidal em um sistema com $500 \mathrm{~h}$ de envelhecimento térmico [66, 67]. Por agitação, o sistema trifásico se homogeniza em sol monofásico instável que, após repouso, retorna ao sistema trifásico.

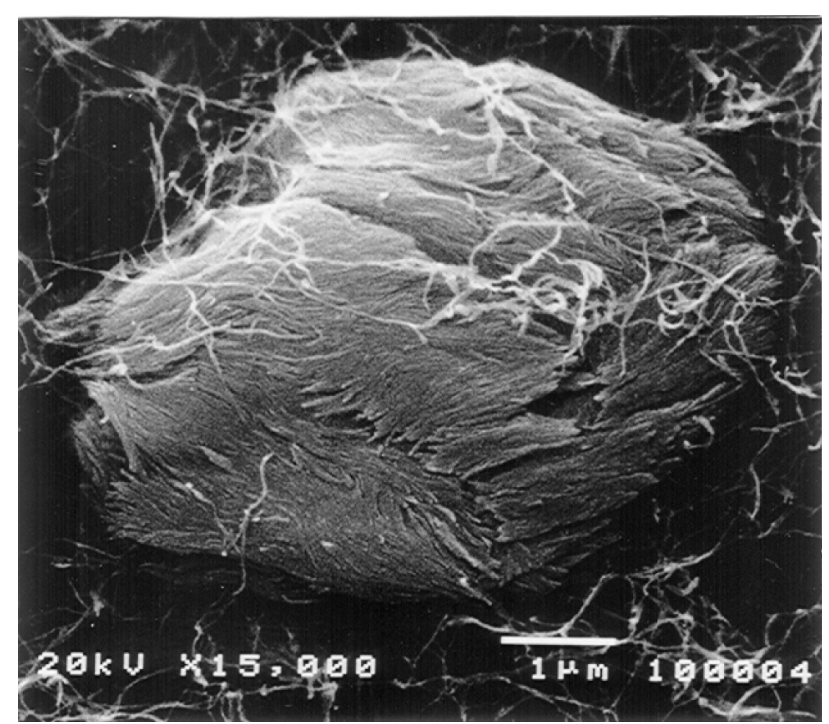

Figura 10: Micrografia obtida por microscopia eletrônica de varredura de um cristal coloidal constituído pela ordenação de fibrilas de "Pseudoboemita" depositadas a partir de um sol envelhecido por $500 \mathrm{~h}$ a $90{ }^{\circ} \mathrm{C}[66,67]$.

[Figure 10: SEM micrograph of a colloidal crystal formed by tridimensional ordination of pseudoboehmite fibrils deposited from a sol aged for $500 \mathrm{~h}$ at $\left.90^{\circ} \mathrm{C}[66,67].\right]$

Continuando o aquecimento de $500 \mathrm{~h}$ até $1000 \mathrm{~h}$, quando as observações foram encerradas, observa-se o seguinte: a) o material de cor branca aumenta em quantidade com o tempo de envelhecimento e deposita; b) desaparece a separação em duas fases liquidas e tem-se uma fase liquida, que é um sol turvo, de cor branca, com aspecto leitoso; c) sóis monofásicos com $1000 \mathrm{~h}$ de envelhecimento térmico a $90{ }^{\circ} \mathrm{C}$ passam a ter 8 a $9 \mathrm{~g} / \mathrm{L}$ de $\mathrm{AlOOH}$. A Fig. 11 é o resultado de microscopia eletrônica de transmissão do sol monofásico com $1000 \mathrm{~h}$ de envelhecimento térmico, mostrando os feixes de fibrilas. A Fig. 12 é o resultado da microscopia eletrônica de varredura da superfície de uma

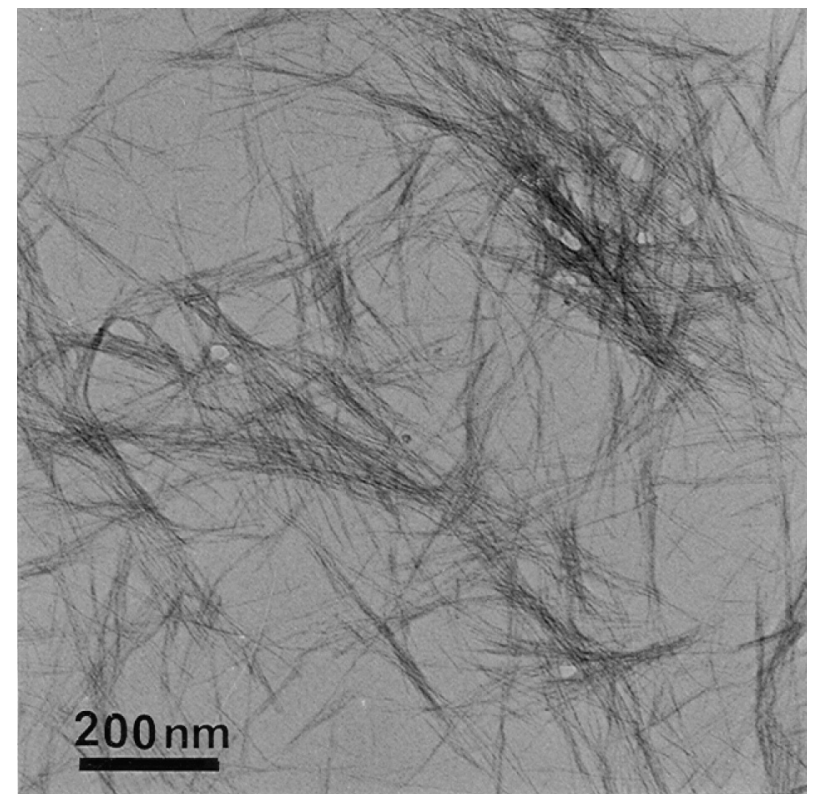

Figura 11: Micrografia obtida por microscopia eletrônica de transmissão das fibrilas do sol monofásico após $1000 \mathrm{~h}$ de envelhecimento térmico a $90{ }^{\circ} \mathrm{C}$, mostrando aglomeração das fibrilas em feixes.

[Figure 11: Transmission electron microscopy micrograph of the fibrils from the monolayer sol after $1000 \mathrm{~h}$ of thermal aging at $90^{\circ} \mathrm{C}$, showing fibril agglomeration into bundles.]

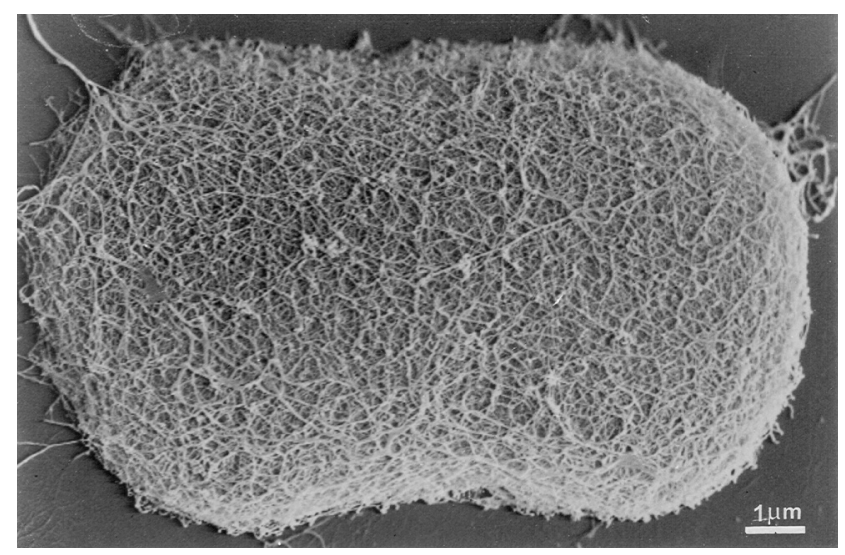

Figura 12: Micrografia obtida por microscopia eletrônica de varredura da superfície de uma gota do sol monofásico com $1000 \mathrm{~h}$, congelada e seca, mostrando o arcabouço tridimensional formado pelas fibrilas [66, 67].

[Figure 12: SEM micrograph of the surface of a microdrop of the monolayer sol, $1000 \mathrm{~h}$ aged, frozen and dried, showing the tridimensional network of the fibrils in the sol [66, 67].]

microgota, congelada e seca pelo método do ponto critico, mostrando o arcabouço tridimensional formado pelo feixe de fibrilas do sol de $1000 \mathrm{~h}$ de envelhecimento térmico a $90^{\circ}[78,79]$. A organização tridimensional das fibrilas dispersas e em cristais nos três tipos foi descrita em detalhe por microscopia eletrônica de transmissão e microscopia eletrônica de varredura por Faria et al. [66].

Viscosidade e algumas aplicações dos sóis - Todos os sóis, com qualquer idade de envelhecimento térmico, 
apresentaram viscosidade tixotrópica. Foi observado que os sóis contendo $70 \mathrm{~g} / \mathrm{L}$ de $\mathrm{AlOOH}$, a partir de $8 \mathrm{~h}$ de envelhecimento a $90{ }^{\circ} \mathrm{C}$, apresentavam valor da viscosidade aparente de 10 a $15 \mathrm{cP}$ (m.Pa.s) e que esse valor aumentava rapidamente até $48 \mathrm{~h}$, quando atingiu 100 a $105 \mathrm{cP}$; continuando o aquecimento até cerca de $192 \mathrm{~h} / 200 \mathrm{~h}$, a viscosidade aparente atinge o valor máximo, que é da ordem de 780/800 cP; continuando o aquecimento até $500 \mathrm{~h}$, a viscosidade diminui desde $200 \mathrm{~h}$ até valores da ordem de 5 a $7 \mathrm{cP}$ para $500 \mathrm{~h}$ de aquecimento do sol monofásico. Essa variação da viscosidade do sol de $70 \mathrm{~g} / \mathrm{L}$ com o tempo pode ser interpretada como: a) o aumento da viscosidade até um valor máximo após $200 \mathrm{~h}$ de aquecimento é devido ao aumento do comprimento das fibrilas; b) admitindo que as fibrilas cresçam linearmente por polimerização por adição, tipo olação ou oxolação [68-70], a $200{ }^{\circ} \mathrm{C}$ a polimerização pára e as fibrilas param de crescer; c) com a continuação do aquecimento por mais $200 \mathrm{~h}$, a diminuição da viscosidade é devida à diminuição do teor de fibrilas, causada pela formação e precipitação dos cristais coloidais da Fig. 12.

É importante assinalar o fato de que a rápida elevação da viscosidade nas primeiras $48 \mathrm{~h}$ de aquecimento no sol de 70 $\mathrm{g} / \mathrm{L}$ de $\mathrm{AlOOH}$ ocorre também se a concentração for duas ou três vezes maior; nesses casos forma-se uma pasta espessa que trava o agitador. Portanto, para se obter sóis com cerca de $20 \%$ de $\mathrm{AlOOH}$ [50] para emprego em processo solgel [19], inclusive com nucleação isoestrutural de $\gamma-\mathrm{Al}_{2} \mathrm{O}_{3}$ [30], a concentração deve ser feita pela evaporação da água, porém evitando o aumento do comprimento das fibrilas e a formação dos cristais coloidais de pseudoboemita fibrilar".

Para o emprego em cerâmica, catalisadores e ab/ adsorventes, os sóis de 24 h e 48 h são os recomendados. A variação de área especifica dessas aluminas de transição a partir de sóis de $24 \mathrm{~h}, 48 \mathrm{~h}$ e $168 \mathrm{~h}$ com a temperatura até a formação da $\gamma-\mathrm{Al}_{2} \mathrm{O}_{3}$ está descrita $[35,71]$. Um fato interessante a ser assinalado é o de que as áreas específicas das fibrilas de sóis secos como membranas, tendo 24, 48 e 168 h de envelhecimento a $90^{\circ} \mathrm{C}$, foram medidas pelo método BET de adsorção de nitrogênio $[35,71]$. Os valores obtidos para as fibrilas foram $313 \mathrm{~m}^{2} / \mathrm{g}(24 \mathrm{~h}), 270 \mathrm{~m}^{2} / \mathrm{g}(48 \mathrm{~h})$ e $294 \mathrm{~m}^{2} / \mathrm{g}$ $(168 \mathrm{~h})$; isso significa que são "high surface materials" [36], porque a área de superfície específica é maior que $100 \mathrm{~m}^{2} / \mathrm{g}$. A seguir, os diâmetros médios dos poros foram calculados para as três idades de fibrilas e os valores obtidos foram 24

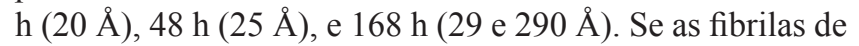
pseudoboemita fosse tubos ocos com diâmetros de $\sim 36 \AA$, esses diâmetros de poros poderiam ser interpretados como diâmetros internos dos tubos das fibrilas de pseudoboemita. Essa é uma hipótese a ser estudada com maiores detalhes. Sóis com $168 \mathrm{~h}$ de aquecimento, bifásicos permitem produzir membranas assimétricas e esferas caneladas na superfície, constituídas de pseudoboemita fibrilar ou por $\gamma-\mathrm{Al}_{2} \mathrm{O}_{3}$, que são eficientes para a fixação e o crescimento de microorganismos, especialmente levedura de cerveja [72]. A comparação entre fases de transformação térmica de pseudoboemita fibrilar com $168 \mathrm{~h}$ de envelhecimento e microcristais de boemita $\mathrm{BC}$ sintética hidrotermicamente com a morfologia de ripas estão descritas [73].

\section{CONCLUSÕES}

Podem ser preparados sóis aquosos de pseudoboemita fibrilar, contendo $70 \mathrm{~g} / \mathrm{L}$ de $\mathrm{AlOOH}$, pela reação entre alumínio em pó e solução aquosa de ácido acético. Esses sóis são preparados aquecendo os produtos da reação $\mathrm{Al}(\mathrm{OH})_{3} \mathrm{e}$ $\mathrm{Al}(\mathrm{OH})_{2} \mathrm{CH}_{3} \mathrm{COO}$ a $90{ }^{\circ} \mathrm{C} \pm 2{ }^{\circ} \mathrm{C}$, a volume constante, por tempo variando de $24 \mathrm{~h}$ a $192 \mathrm{~h}$. As partículas constituintes desses sóis com diversas idades de envelhecimento são de hidróxido de alumínio cristalino pseudoboemita; elas são estáveis quanto à dissolução em água, recristalização em trihidróxidos de alumínio cristalinos. As partículas ou cristais desses sóis são fibrilas, as fibrilas são flexíveis e seu comprimento aumenta com o tempo de envelhecimento térmico. Exemplificando: 0,53 nm após $24 \mathrm{~h}, 450 \mathrm{~nm}$ após $168 \mathrm{~h}$ e $720 \mathrm{~nm}$ após $192 \mathrm{~h}$.

Os sóis aquosos apresentam viscosidade tixotrópica crescente até um valor máximo após $192 \mathrm{~h}$ de envelhecimento térmico a $90^{\circ} \mathrm{C}$. Os sóis até $72 \mathrm{~h}$ de envelhecimento, deixados em repouso à temperatura ambiente, são monofásicos. Os sóis com mais de 72 h até 192 h são bifásicos, se deixados em repouso; retornam a sóis monofásicos instáveis por agitação, que voltam a bifásico com o repouso. Os sóis de $24 \mathrm{~h}$ e $48 \mathrm{~h}$ são recomendáveis para usos em cerâmica avançada (processo sol/gel nucleado), ab-adsorventes; catálise; aquelas de $168 \mathrm{~h}$ para membranas assimétricas para fixação e suporte de crescimento de microorganismos, como levedura de cerveja.

\section{AGRADECIMENTOS}

Os autores agradecem à Química Valquiria F. J. Kozievitch pela ajuda na elaboração do manuscrito.

\section{REFERÊNCIAS}

[1] I. Valeton, Bauxites, Elsevier, Amsterdam, Holanda (1972) 62.

[2] W. D. Keller, J. F. Westcott, A. O. Bledsol, Clays Clay Min. 2 (1953) 7.

[3] W. H. Gitzen, Alumina as a Ceramic Material, Am. Ceram. Soc., Columbus, Ohio, EUA (1970) 4.

[4] B. C. Lippens, J. J. Steggerda, Active Alumina, em B. G. Linsen, Physical and Chemical Aspects of Adsorbents and Catalysts, Academic Press, London, Inglaterra (1970) 180.

[5] K. Wefers, C. Misra, Oxides and Hydroxides of Aluminium, Alcoa Labs., Alcoa Center, Pennsylvania, EUA (1987) 50.

[6] D. Papée, R. Tertian, R. Biais, Bull. Soc. Chim. France 5 (1958) 1301.

[7] O. Krivoruchko, R. A. Buyanov, N. A. Fedorov, L. M. Plyasova, Russian J. Inorg. Chem. 23 (1978) 988.

[8] R. Tettenhorst, D. A. Hofmann, Clays Clay Min. 24 (1980) 373.

[9] H. Souza Santos, A. Vallejo-Freire, P. Souza Santos, 
Kolloid Zeit. 133 (1953) 101.

[10] J. Bugosh, Fibrous Alumina Monohydrate and its Production, U.S. Patent 2,915,475 (Dec. 1, 1959).

[11] R. K. Iler, J. Am. Ceram. Soc. 44 (1961) 618.

[12] 1. Abrams, M. J. D. Low, I \& EC Product Research Devel. 8 (1969) 38.

[13] C. Misra, Industrial Alumina Chemicals, Am. Chem. Soc. Monograph 184, Washigton, EUA (1986) 48.

[14] T. A. Rings, Fundamentals of Ceramic Powder Processing and Synthesis, Academic Press, San Diego, EUA (1986) 36.

[15] P. Souza Santos, P. K. Kiyohara, H. Souza Santos, Bol. Técn. Petrobrás 41 (1998) 45.

[16] G. H. Schiroky, D. E. Day, Am. Ceram. Soc. Bull. 59 (1980) 718.

[17] S. Jagota, R. Raj, J. Crystal Growth 85 (1987) 527.

[18] J. Sappei, D. Goeuriot, F. Thevenot, Y. Laurent, J. Guyader, P. L. Haridon, J. Eur. Ceram. Soc. 8 (1991) 257.

[19] D. S. Horn, G. L. Messing, J. Am. Ceram. Soc. 72 (1989) 1719.

[20] A. Y. Chen, J. D. Cawley, J. Am. Ceram. Soc. 75 (1992) 575.

[21] I. P. Kilbride, A. J. Barker, Brit. Ceram. Trans. 93 (1994) 187.

[22] S. Ananthakumar, A. R. R. Menon, K. Prabhakaran, K. G. K Warrier, Ceram. Int. 27 (2001) 231.

[23] A. C. Pierre, D. R. Ulmann, Mater. Res. Soc. Symp. Proc. 32 (1984) 119.

[24] K. Strenge, U. Bollmann, Colloids Surfaces 57 (1991) 139.

[25] D. Mishra, S. Anand, R. K. Panda, Mater. Letters 42 (2000) 38.

[26] A. R. Boccaccini, C. Kaya, Ceram. Int. 28 (2002) 893

[27] R. Petrovic, S. Milonjic, V. Jokanovic, L. KosticGvozdenovic, I. Petrovic-Prelevic, D. Janackovic, Powder Technol. 133 (2003) 185.

[28] S. J. Wilson, Miner. Mag. 43 (1979) 301; J. Sol. State Chem. 30 (1979) 247.

[29] S. J. Wilson, M. H. Stacey, J. Coll. Interf. Sci 82 (1981) 507.

[30] M. Kuragai, G. L. Messing, J. Am. Ceram. Soc. 68 (1985).

[31] H. Souza Santos, P. Souza Santos, Mater. Lett. 13 (1992) 175.

[32] H. Souza Santos, P. K. Kiyohara, P. Souza Santos, Ceram. Int. 20 (1994) 175

[33] P. Souza Santos, P. K. Kiyohara, H. Souza Santos, Mater. Res. Bull. 37 (1996) 799.

[34] V. F. J. Kozievitch, G. A. Rocha, W. S. Maia, H. Souza Santos, P. K. Kiyohara, A. C. V. Coelho, P. Souza Santos, Mater. Sci. Forum 498/499 (2005) 658.

[35] A. C. V. Coelho, G. A. Rocha, P. Souza Santos, H. Souza Santos, P. K. Kiyohara, Revista Matéria 13 (2008) 329.

[36] R. K. Oberlander, Aluminas for Catalysts - Their Preparation and Properties, in B. E. Leach, Appl. Ind. Catalysis, vol.3, Academic Press, Orlando, Florida, EUA (1984) 63.
[37] I. I. M. Tijburg, J. W. Gens, H. W. Zandbergen, J. Mater. Sci. 26 (1991) 6479.

[38] T. L. Hong, H. T. Liu, C. T. Yeh, S. H. Chen, F. C. Sheu, L. J. Lev, C. I. Wang, Appl. Catalysis A: General 158 (1997) 257, citando: T. Y. Chou, C. H. Leu, C. T. Yeh, Catal. Today 26 (1995) 53.

[39] A. F. M. Leenaars, K. Keizer, A. J. Burggraff, J. Mater. Sci. 19 (1984) 1077.

[40] B. Djuricic, S. Pickering, D. McGarry, P. Tambuyser, P. Thomas, J. Mater. Sci. 34 (1999) 1911.

[41] J. Li, X. Wang, L. Wang, Y. Hao, Y. Huang, Y. Zhang, X. Sun, X. Liu, J. Membrane Sci. 275 (2006).

[42] R. A. Shelleman, G. L. Messing, M. Kumagai, J. NonCryst. Solids 82 (1986) 277.

[43] R. A. Shelleman, G. L. Messing, J. Am. Ceram. Soc. 71 (1988) 317.

[44] X. Yang, A. C. Pierre, D. R. Uhlmann, J. Non-Cryst. Solids 100 (1988) 371.

[45] L. Pach, R. Roy, S. Komarnemi, J. Mater. Res. 5 (1990) 278.

[46] Z. Hrabe, S. Komarnemi, L. Pach, R. Roy, J. Mater. Res. 7 (1992) 444.

[47] Z. Hrabe, O. M. Spaldon, L. Pach, J. Kozankova, Mater. Res. Bull. 27 (1992) 397.

[48] E. Morgado, Y. L. Lam, S. M. Menezes, L. F. Nazar, J. Coll. Interf. Sci. 176 (1995) 432.

[49] L. Baca, J. Plewa, L. Pach, J. Opfermann, J. Thermal Anal. Cal. 66 (2001) 803.

[50] J. D. F. Ramsay, S. R. Daish, C. J. Wright, Disc. Faraday Soc. 62 (1982) 65.

[51] T. P. Meloy, Physicochemical Properties Relating to Shape, in J. K. Beddow, T. P. Meloy, Advanced Particulate Morphology, CRC Press, Boca Raton, Florida, EUA (1997) 97.

[52] C. Orr, Physical Characterization Techniques for Particles, in G. Y. Onoda, L. L. Hench, Ceramic processing before firing, John Wiley, Chichester, EUA (1978) 49.

[53] D. W. Richerson, Modern Ceramic Engineering, 2 ${ }^{\text {a Ed., }}$ Marcel Dekker, New York, EUA (1992) 447.

[54] J. Y. C. Ching, L.C. Klein, J. Am. Ceram. Soc. 71 (1988) 83.

[55] J. Y. C. Ching, L.C. Klein, J. Am. Ceram. Soc. 71 (1988) 86.

[56] W. L. Davidson, X-Ray diffraction methods as applied to powders and metals, em W.G. Berl, Physical Methods in Chemical Analysis, vol 1, Academic Press, New York, EUA (1950) 66.

[57] G. W. Brindley, T.C. Simonton, Clays Clay Min. 32 (1984) 235.

[58] C. Tchoubar, A. Oberlin, J. Microscopie 2 (1963) 415.

[59] D. Aldocroft, G. C. Bye, C. A. Hughes, J. Appl. Chem. 19 (1969) 167.

[60] W. J. McHardy, A. P. Thompson, Miner. Mag. 38 (1972) 358.

[61] H. Elderfield, J. D. Hem, Miner. Mag. 39 (1973) 89.

[62] G. C. Bye, J. G. Robinson, J. Appl. Chem. Biotechn. 24 (1974) 633. 
[63] C. J. Cerna, J. L. White, S. L. Hem, Clays Clay Min. 25 (1977) 384.

[64] H. Freundlich, Colloid Capillary Chemistry, Dutton, New York, EUA (1992) 390.

[65] H. Freundlich, Sols with Non-Spherical Particles, em H. N. Holmes, Colloid Symp. Monograph, n. 2, Chemical Catalog, New York, EUA (1925) 46.

[66] F. P. Faria, "Arranjo tridimensional de fibrilas em sóis de pseudoboemita observado por técnicas de criofratura e de secagem pelo método do ponto crítico", Diss. Mestrado, Dep. de Eng. Química, Escola Politécnica da Universidade de S. Paulo, S. Paulo, SP (1994).

[67] F. P. Faria, P. Souza Santos, H. Souza Santos, Mater. Chem. Phys. 76 (2002) 267.

[68] C. L. Rollison, Anion Penetration, in J. Bailar, The Chemistry of Coordination Compounds, John Wiley, New
York, EUA (1958) 459.

[69] P. Souza Santos, "Estudo, ao microscópio eletrônico, da precipitação e do envelhecimento de hidróxido de alumínio coloidal", Tese de Doutoramento, Dep. Eng. Química, Escola Politécnica da Universidade de S. Paulo (1958).

[70] J. P. Jolivet, Metal Oxide Chemistry and Synthesis, John Wiley, Chichester, EUA (2000) 75.

[71] G. A. Rocha, "Estudo comparativo entre aluminas preparadas a partir de diferentes precursores de boemita", Diss. Mestrado, Dep. Engenharia Metalúrgica e de Materiais, Escola Politécnica da Universidade de S. Paulo, S. Paulo, SP (2004)

[72] P. K. Kiyohara, U. A. Lima, H. Souza Santos, P. Souza Santos, Braz. J. Microbiology 34 (2003) 129.

[73] H. Souza Santos, Acta Microscópica 7 (1998) 1.

(Rec. 25/04/2008, Ac. 15/08/2008) 